\title{
What Limits Cardiac Performance during Exercise in Normal Subjects and in Healthy Fontan Patients?
}

\author{
André La Gerche ${ }^{1,2}$ and Marc Gewillig ${ }^{1}$ \\ ${ }^{1}$ University Hospital, Catholic University of Leuven, 3000 Leuven, Belgium \\ ${ }^{2}$ St Vincent's Hospital, University of Melbourne, 3065 Fitzroy, Australia \\ Correspondence should be addressed to Marc Gewillig, marc.gewillig@uzleuven.be
}

Received 16 February 2010; Revised 11 May 2010; Accepted 27 July 2010

Academic Editor: Patricia A. Nixon

Copyright ( 2010 A. La Gerche and M. Gewillig. This is an open access article distributed under the Creative Commons Attribution License, which permits unrestricted use, distribution, and reproduction in any medium, provided the original work is properly cited.

\begin{abstract}
Exercise is an important determinant of health but is significantly reduced in the patient with a univentricular circulation. Normal exercise physiology mandates an increase in pulmonary artery pressures which places an increased work demand on the right ventricle $(\mathrm{RV})$. In a biventricular circulation with pathological increases in pulmonary vascular resistance and/or reductions in RV function, exercise-induced augmentation of cardiac output is limited. Left ventricular preload reserve is dependent upon flow through the pulmonary circulation and this requires adequate RV performance. In the Fontan patient, the reasons for exercise intolerance are complex. In those patients with myocardial dysfunction or other pathologies of the circulatory components, it is likely that these abnormalities serve as a limitation to cardiac performance during exercise. However, in the healthy Fontan patient, it may be the absence of a sub-pulmonary pump which limits normal increases in pulmonary pressures, trans-pulmonary flow requirements and cardiac output. If so, performance will be exquisitely dependent on pulmonary vascular resistance. This provides a potential explanation as to why pulmonary vasodilators may improve exercise tolerance. As has recently been demonstrated, these agents may offer an important new treatment strategy which directly addresses the physiological limitations in the Fontan patient.
\end{abstract}

\section{Introduction}

Exercise is an important determinant of health and provides numerous cardiovascular, psychological, and prognostic benefits [1]. The Fontan operation, and its refinements, have proved a major success with a majority of patients with univentricular malformations now surviving into adulthood with a good quality of life [2]. However, exercise tolerance is significantly reduced and the factors responsible for this exertional limitation are incompletely understood. This paper aims to provide a unique perspective on the limitations of the Fontan circulation by focusing on exercise constraints in a normal biventricular circulation. Rather than describing abnormalities within the components of a Fontan circulation, we will focus on highlighting the importance of that which is missing-a prepulmonary pump. Popular models of exercise physiology have concentrated on the systemic circulation and the left ventricle (LV) as the primary determinant of cardiac output (CO) augmentation during physical exertion [3]. These models are likely to be accurate in patients with heart failure. However, in the healthy subject, there is a large reserve for exercise-induced augmentation of the systemic ventricle and vasculature. A less commonly cited determinant of cardiac performance during exercise is the "lesser circulation" of the RV and the pulmonary circulation [4-6]. We will discuss the evidence supporting the concept that the right ventricle (RV) is a determinant of exercise performance and that, in its absence, pulmonary vascular resistance becomes a critical limitation. The recent availability of relatively specific pulmonary vasodilators has given these physiological discussions immediate clinical relevance. The potential benefit of these agents [7] is consistent with physiological theory and represents an exciting avenue of investigation in Fontan patients.

\section{Cardiac Output Augmentation during Exercise in the Normal Heart}

Exercise performance is defined by the ability of the body's working muscles to utilize oxygen, and this can 
be measured by $\mathrm{VO}_{2}$ max on cardiorespiratory testing. It has been demonstrated that the skeletal muscles' ability to utilize oxygen far exceeds the capacity of the cardiovascular system to deliver oxygen [8]. Thus, cardiac output (CO) becomes the limiting step, and explains $70 \%-85 \%$ of the variance in $\mathrm{VO}_{2}$ max with the remainder being determined by peripheral factors such as mitochondrial density which enables greater oxygen extraction [9]. In healthy subjects, CO may be expected to increase 3 - to 5 -fold whilst increases are even greater in athletes. Cardiac output is enhanced by (1) greater preload, (2) increased heart rate, (3) increased myocardial contractility, and (4) reduced afterload during exercise, and both ventricles need to generate the same stroke volume. Thus we can speculate as to what is the "weak link" by asking which factor is closest to its physiological limit during exercise. Conventional teaching is that it is the $L V$ and its interaction with the systemic circulation which determines cardiac output. However, LV functional measures and measures of systemic vascular tone have not been shown to predict exercise performance as might be expected if they were the limitation of exercise performance [10-12].

2.1. Preload and Exercise. Preload may be defined as the passive stretch applied to the myocardium prior to the initiation of active contraction. According to the FrankStarling mechanism, increases in preload augment cardiac output. The extent to which this mechanism contributes to exercise-induced $\mathrm{CO}$ augmentation remains a point of debate. Studies using radionuclide ventriculography have reported large increases in LV end-diastolic volume during exercise and minimal or no decrease in LV end-systolic volume. Warburton et al. [13, 14] suggested that this increase in LV preload was the dominant means by which $\mathrm{CO}$ increased during exercise, especially in well-trained athletes [13, 14]. However, more recent exercise studies using 2D echocardiography [3,15-19] and cardiac magnetic resonance (CMR) [20-22] have found little or no increase in LV end-diastolic volume and that augmentation of stroke volume is primarily due to decreases in LV end-systolic volume. Assimilating these contrasting findings is difficult, but perhaps a reasonable summary may be that enhanced LV preload contributes to, but is not the sole determinant of, exercise-induced increases in stroke volume.

Accepting the contribution of LV preload in cardiac output augmentation, the question arises as to what determines $\mathrm{LV}$ preload? This is seldom discussed in the literature. It is often assumed that the LV generates flow which then serves as its own preload with, perhaps, some facilitation from skeletal muscle acting to enhance venous return. With a few notable exceptions $[4-6,23]$, the RV and pulmonary circulation have rarely been considered as potential constraints on LV filling in normal exercise physiology. We speculate that its importance may have been underappreciated. At least in settings where RV afterload is increased, reduced flow in the presystemic circulation can result in reduced LV preload and, thus, cardiac output limitation. Holverda et al. $[20,21]$ demonstrated reduced LV filling during exercise in patients with pulmonary hypertension and obstructive airways disease. Also, the demonstration of improved exercise tolerance with pulmonary vasodilators provides some indirect affirmation of this concept. It has been shown that sildenafil $[24,25]$ and bosentan [26] can increase exercise performance at altitude or during hypoxic exercise although no benefit has been demonstrated in normoxic settings. It was concluded by Faoro et al. [26] that the pulmonary circulation provides a limitation to cardiac output when there is hypoxic pulmonary vasoconstriction. The lack of efficacy of selective pulmonary vasodilators during normoxic exercise may suggest that pulmonary vasodilation is close to maximal in this setting [27].

Frequently, exercise intolerance is attributed to diastolic dysfunction, and it is implied that the LV filling impairment is due to pathology of the LV myocardium. However, it is important to note that it is extremely difficult to differentiate impaired filling due to myocardial stiffness from that due to reduced preload when using noninvasive measures [28]. Echocardiographic measures are similar when there is a lack of "push" from reduced preload and when there is a lack of "suck" from LV impairment. Puwanant et al. described reductions in traditional and novel diastolic measures in patients with pulmonary hypertension despite relatively normal LV function [28]. Hart et al. reported measures of diastolic dysfunction which were associated with preload reduction following marathon running. These changes normalized immediately with preload augmentation by leg lifting [29]. Finally, in a large cohort of patients with obstructive pulmonary disease, Barr et al. attributed impaired LV filling to preload reduction through increased pulmonary vascular resistance rather than myocardial stiffness resulting from hypoxia or hypertension [30].

2.2. Afterload and Exercise. Afterload can be defined as the myocardial stress achieved during contraction, the major determinants being systolic pressure, cavity size, and wall thickness [31]. Decreases in LV afterload serve to increase stroke volume and this has been emphasized as an important source of cardiac output modulation in popular models of exercise physiology. It has even been suggested that the exercise-induced decreases in systemic vascular resistance (due to vasodilation in skeletal muscle) is the primary determinant of $\mathrm{CO}$ augmentation, and that the resultant increases in heart rate and contractility are simply a compensation to maintain systemic blood pressures $[32,33]$. However, it has been well established that the capacity for peripheral vasodilation far exceeds the capacity for cardiac output augmentation, implying that the limitation to cardiac output is upstream of the peripheral circulation [34].

Characterization of RV afterload during exercise is more difficult because its measurement requires invasive pulmonary artery catheterization or echocardiographic estimates, both of which can be challenging during exercise. Whilst it is commonly stated that pulmonary artery pressures increase only slightly with exercise $[35,36]$, multiple studies have reported considerable increases in pulmonary artery pressures [4, 23, 37-42]. Kovacs et al. [42] combined data from nearly 200 patients in invasive studies to demonstrate that mean pulmonary artery pressures increased in a linear 
TABle 1: Comparison of exercise-induced changes in pulmonary versus systemic vascular resistance and pressure. The pulmonary vasculature has very low resistance at rest but relatively limited capacity for further reduction. Therefore, exercise-induced increases in flow result in greater pressures due to the inability to compensate by reducing resistance. As described by the simplified Poiseuille's law: Pressure $=$ Flow $\times$ Resistance.

\begin{tabular}{|c|c|c|}
\hline \multicolumn{3}{|c|}{ Left Ventricle Right Ventricle } \\
\hline \multicolumn{3}{|l|}{ Rest } \\
\hline Cardiac Output (L/min) & 5 & 5 \\
\hline Vascular resistance $\left(\right.$ dyne-sec $\left.\cdot \mathrm{cm}^{5}\right)$ & 1100 & 70 \\
\hline Afterload Pressure (mmHg) & $130 / 75(85)$ & $25 / 9(15)$ \\
\hline \multicolumn{3}{|c|}{ Exercise } \\
\hline Cardiac Output (L/min) & 25 & 25 \\
\hline Vascular resistance (dyne-sec $\cdot \mathrm{cm}^{5}$ ) & $\downarrow \downarrow$ & $\downarrow$ \\
\hline Afterload Pressure (mmHg) & $\uparrow$ & $\uparrow \uparrow \uparrow$ \\
\hline
\end{tabular}

manner with exercise which explains why relatively high pressures have been measured in those with good exercise tolerance. Argiento et al. [37] described exercise-induced increases in systolic pulmonary artery pressures that frequently exceeded $60 \mathrm{mmHg}$ in healthy subjects whilst Bidart et al. [39] reported even greater increases in athletes. It may be that these increases in pulmonary artery pressures are a result of an inability for the pulmonary vasculature to reduce its resistance sufficiently to compensate for the increased CO of exercise. This concept is best summarized by the simplified Poiseuille's relation in which CO is proportional to pressure and inversely to resistance [3]. Pulmonary vascular resistance is very low at rest and previous studies have suggested that its capacity for reduction is only $20 \%-50 \%[23,35,43]$. This represents a marked difference to the systemic circulation where greater reductions in peripheral resistance moderate increases in LV afterload (see Table 1).

2.3. Contractility and Exercise. Contractility is an intrinsic property of the myocardium, is load independent, and is most frequently derived from invasive indices such as the slope of the end-systolic pressure volume relation or the preload recruitable stroke work [44]. There are no studies which have assessed and compared LV and RV contractility during exercise in healthy subjects. At rest, RV measures of mass [45] and contractility [46] are one-third to onefifth those of the LV and this appropriately matches the pressure requirements of each [47]. However, when RV afterload increases, RV contractility may be unable to maintain ventriculo-arterial coupling such that cardiac output declines. MacNee [48] described marked reductions in RV stroke volume $(\sim 30 \%)$ when pulmonary artery pressures doubled and compared this with only slight decreases in LV stroke volume with physiological increases in mean arterial pressures. During exercise, Morrison et al. [23] used firstpass radionuclide ventriculography and invasive pressure measures to demonstrate a progressive improvement in RV ejection fraction with exercise-induced reductions in pulmonary vascular resistance. However, in comparison with the systemic circulation, increases in pulmonary vascular pressures were relatively greater, and reductions in vascular resistance were less. This would suggest that exercise results in a disproportionate workload for the RV, a concept which is further supported by the demonstration that cardiac fatigue predominantly affects the RV after intense prolonged exercise $[49,50]$. Therefore, there are reasonable grounds to contend that exercise-induced increases in RV load represent a potential for cardiac output limitation. For cardiac output to be maintained during exercise, the RV must greatly augment its contractility, possibly more so than the LV. As will be discussed, this is problematic for exercise performance when $\mathrm{RV}$ function is reduced or absent.

2.4. Heart Rate and Exercise. Heart rate is a major determinant of cardiac output, particularly in the later phases of exercise when stroke volume augmentation plateaus [3]. It is, however, inefficient for the heart rate to increase such that LV filling is impaired. Thus, although the factors which determine maximal heart rate during exercise are incompletely understood, it is likely that cardiopulmonary baroreceptor activation contributes to maintaining an appropriate balance between LV preload and heart rate during exercise [51].

\section{The Healthy Fontan Patient and Exercise: How Is Reduced Exercise Capacity Explained?}

In the Fontan circulation, the systemic and pulmonary vascular beds are connected in series without the presence of a pre-pulmonary pump to add forward energy to flow through the lungs (Figure 1). Typically $\mathrm{CO}$ in a Fontan circulation at rest is decreased to $70 \%$ (range 50\%-80\%) of normal and an increase in peripheral oxygen utilization compensates for this reduction in oxygen supply [52]. During exercise, oxygen utilization remains normal or supranormal but maximal $\mathrm{CO}$ is approximately half that of normal [53]. Control of cardiac output in a Fontan circulation is complex with a different interplay of contractility, afterload, preload, and heart rate [54]. The implications of exercise on each of these variables will be discussed and contrasted with that already described for normal subjects.

3.1. Preload and Exercise in the Fontan Patient. There is limited data in which LV filling during exercise has been assessed in the Fontan patient. More frequently, studies have assessed the hemodynamic response to low-dose dobutamine as an exercise surrogate. Senzaki et al. [55, 56] have twice demonstrated limitations in preload reserve in this setting. They demonstrated a significant $25 \%$ reduction in LV end-diastolic pressure in the Fontan patient whereas there was no change in subjects with normal circulation [56]. Using variables derived from ventricular pressure-area hybrid loops, they found that cardiac index was dependent upon preload (end-diastolic area) and not contractility (ventricular elastance) or afterload (arterial elastance). Thus, inadequate preload reserve was implicated in the failure for $\mathrm{CO}$ to augment with $\beta$-adrenergic stimulation. RobbersVisser et al. [57] used CMR imaging to provide a volumetric assessment of dobutamine-induced cardiac augmentation in 


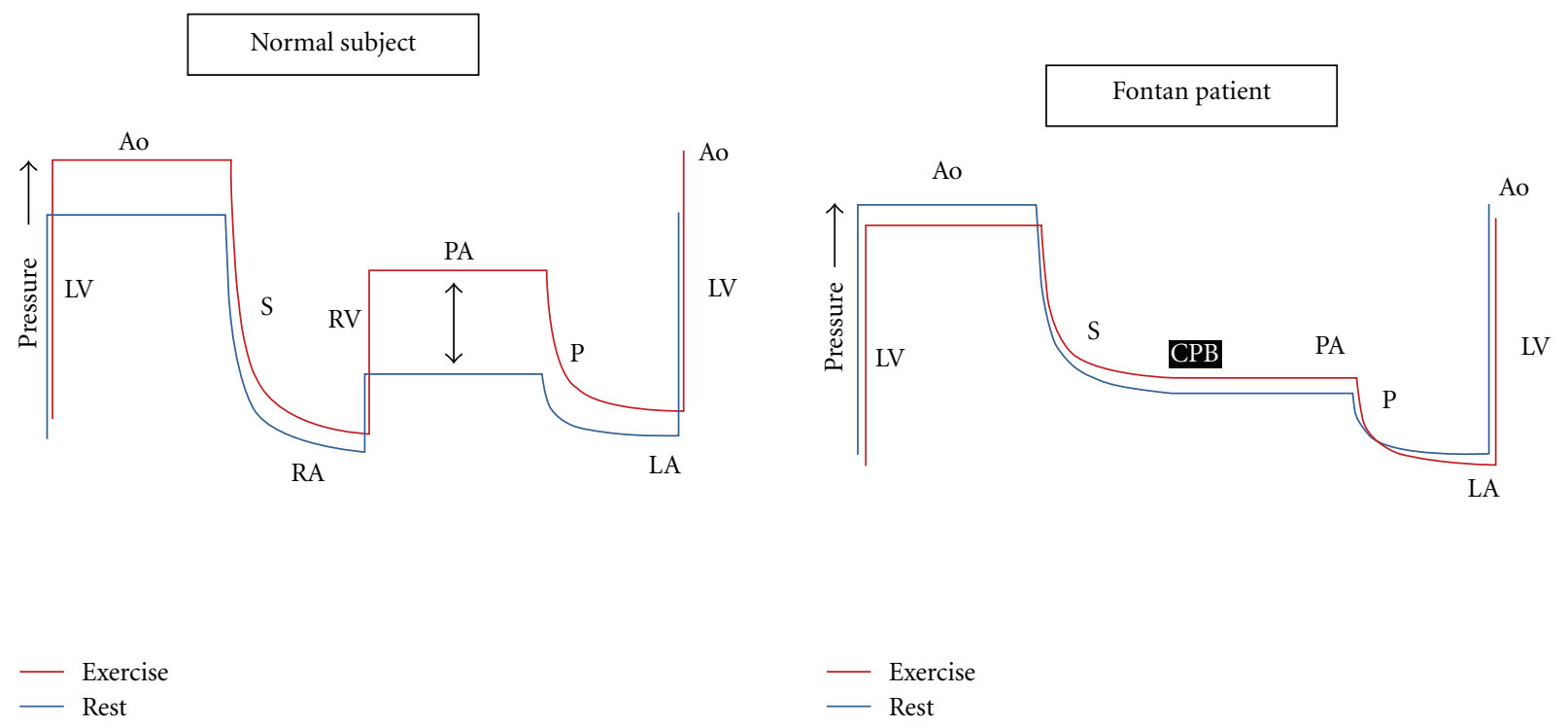

(a)

(b)

FIGURE 1: Theoretical schema to illustrate circulatory pressure changes in normal and Fontan patients at rest and during exercise. In the normal circulation, pressure is generated in the systemic ventricle (LV) to produce flow in the aorta (Ao) and systemic circulation (S). Pressure dissipates across the systemic microcirculation such that right atrial (RA) pressures are low. The pre-pulmonary pump (RV) provides the pressure to generate the flow in the pulmonary artery (PA) which then dissipates in the pulmonary circulation (P) but is sufficient to maintain preload in the left atrium (LA). During exercise, systemic vascular resistance falls such that there is little increase in mean LV pressure requirements. However, more substantial pressure increases are required in the RV (purple arrow), and these pressure requirements increase with exercise intensity. In the Fontan patient (below), the cavopulmonary bypass (CPB) does not provide any contractile force and, therefore, flow through the pulmonary circulation is dependent on the pressure difference between the RA and LA. During exercise, trans-pulmonary flow can only be augmented by reductions in pulmonary vascular resistance. Beyond mild to moderate exercise, pulmonary vasodilation is maximal and flow increases require a pre-pulmonary pump. Without this, pulmonary pressure does not rise, trans-pulmonary flow does not increase, LA pressure (preload) does not increase, and cardiac output cannot supply the metabolic demands of exercise.

a young group of patients with Fontan circulation. They described a modest increase in $\mathrm{CO}$ which was entirely due to increasing heart rate. Stroke volume was unchanged due to the fact that end-diastolic volumes decreased to the same extent as end-systolic volumes. This contrasts with the normal physiological response to dobutamine in which stroke volume increases due to a reduction in end-systolic volume and preserved end-diastolic volumes $[58,59]$. Thus, $\mathrm{CO}$ limitation in the Fontan patient was again attributed to inadequate preload reserve suggesting that the constraint to exercise is "up-stream" of the LV.

As discussed previously, measures of impaired filling should not be regarded as synonymous with LV pathology. Preload reduction and myocardial relaxation impairment will manifest similarly on non-invasive measures such as echocardiography. This is well illustrated in a recent study performed by Goldstein et al. [60] in which echocardiographic indices of diastolic dysfunction were demonstrable in 16 of 28 young Fontan patients. However, hemodynamic data performed in a subset of these patients during exercise demonstrated normal LV filling pressures which did not increase with exercise, a finding which is inconsistent with the raised filling pressures expected with diastolic dysfunction $[61,62]$. Rather, these findings again reinforce the concept of preload inadequacy whereby the normal exerciseinduced augmentation in LV filling pressures [42] is absent in the Fontan patient.

3.2. Afterload and Exercise in the Fontan Patient. In the Fontan patient during exercise, the reduction in systemic vascular resistance is less than in the normal subject [52]. It is likely that this represents a secondary phenomenon which attempts to maintain blood pressure when $\mathrm{CO}$ augmentation is reduced. This is achieved by an increase in sympathetic vasomotor tone [63] but is not always sufficient and BP may still fall in the Fontan patient during exercise [52]. Thus the relatively greater systemic afterload in the Fontan patient is a consequence, rather than cause, of reduced output.

As described earlier, normal exercise is associated with an increase in stroke volume and pulmonary artery pressures. A pre-pulmonary pump is required to generate the pressure and flow which enables adequate LV filling during exercise. Without a pre-pulmonary pump, and without adequate preload, cardiac output cannot increase during exercise. Figure 1 provides a graphic summary of this proposed physiology.

Indirect evidence for our proposition that the pulmonary circulation serves as a limitation to exercise in the Fontan 


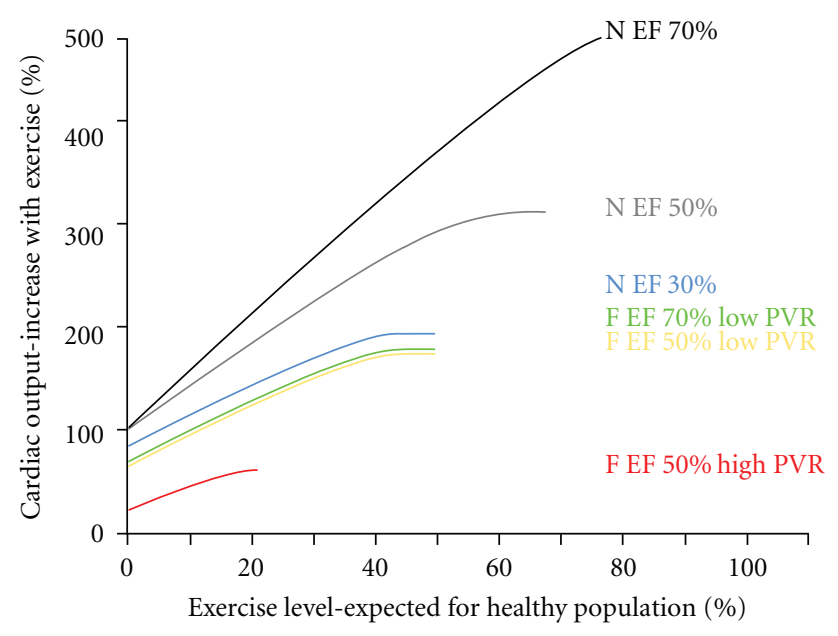

Figure 2: Relationship of output during exercise, pulmonary vascular resistance (PVR), and ventricular function. Cardiac output can increase 5 -fold in a normal $(\mathrm{N})$ subject with a biventricular circuit. If ventricular function is impaired, this will first result in decreased maximal output and subsequently in reduced output at low level of exercise. In Fontan patients (F) output is more influenced by PVR than by ventricular function but all have significantly impaired exercise capacity. EF: ejection fraction.

patient is provided by the demonstration that exercise tolerance is improved with pulmonary vasodilators. Giardini et al. [7] studied the exercise response in 18 Fontan patients before and after a single oral dose of sildenafil as compared with a group of 9 control Fontan patients. An improvement in $\mathrm{VO}_{2} \max (9.4 \pm 5.2 \%)$ and cardiac index $(10.0 \pm 7.2 \%)$ was demonstrable in those who took sildenafil whilst these measures were unchanged in those who did not receive treatment. Pulmonary vasodilator therapy warrants further investigation in Fontan patients given its potential to address a likely source of limitation to exercise.

3.3. Contractility and Exercise in the Fontan Patient. There is limited evidence to suggest that contractility serves as a limitation to $\mathrm{CO}$ in the Fontan patient, with the exception of those patients with advanced ventricular dysfunction [54]. As described earlier, Senzaki et al. [55] used hybrid measures of ventricular elastance to demonstrate that contractility and contractile reserve were largely preserved in the Fontan patient. Studies that have suggested reduced systolic performance in the Fontan ventricle have frequently based conclusions on measures which are load dependent [64]. Furthermore, if abnormalities of the systemic ventricle and the peripheral circulation were a cause of exercise limitation, a benefit in exercise tolerance might be expected with ACE inhibitor therapy but this has not been demonstrated [65]. Figure 2 summarizes the relative contribution of LV function and pulmonary vascular resistance to exercise-induced increases in cardiac output.
3.4. Heart Rate and Exercise in the Fontan Patient. In the Fontan patient, atrial pacing at increased rates does not result in the augmentation of cardiac output that is seen in normal subjects. Rather, there is a proportional reduction in stroke volume which illustrates heart rate has a less direct effect on $\mathrm{CO}$ than in a normal circulation [66]. During exercise, Fontan patients exhibit chronotropic incompetence with a heart rate consistently lower than normal controls, and this has typically been attributed to abnormal reflex control of heart rate or adrenergic dysfunction [67-71]. However, preload inadequacy in the Fontan patient may lead to reducing stroke volume if the ventricular filling time decreases with increasing heart rate. Thus, it is possible that chronotropic incompetence is an adaptive response to prevent hemodynamic compromise in patients with limited preload, such as in the Fontan patient $[72,73]$.

\section{Summary}

Traditional models of exercise physiology have emphasized the performance of the systemic ventricle and systemic circulation. In the Fontan patient with pathology of the systemic ventricle, these abnormalities will largely explain exercise limitation. However, in the healthy Fontan patient, limitation is defined by upstream factors which determine systemic ventricular filling. Inadequate preload reserve has been demonstrated to limit cardiac augmentation caused by ionotropic stimulation and it is likely that this mechanism also explains cardiac limitation during exercise. At rest, pulmonary vascular resistance and pressure are low. During exercise, increases in flow mandate an increase in pressure unless resistance can fall proportionately. Substantial reserve in the systemic circulation moderates pressure increases whilst, in the pulmonary circulation, there is evidence that the capacity for further vasodilation is limited. In a normal biventricular circulation, this results in greater pressure and work demands for the RV during exercise. However, in the Fontan patient, there is no RV to provide this work demand and hence flow, LV filling and CO are unable to increase normally.

This discussion of Fontan physiology has important clinical implications. Treatment regimens are most likely to be efficacious if they address the part of the circulation which serves as the greatest source of limitation. Classical heart failure regimens have demonstrated poor efficacy in Fontan patients but this may be expected if the systemic circulation is not the primary source of limitation. Selective pulmonary vasodilators target a potential "weak link" in the Fontan circuit. Early results with these agents have offered promise and may prove an important means of improving exercise tolerance.

\section{Acknowledgments}

This paper is funded in part by The Eddy Merckx research Fund. André La Gerche receives scholarship funding from the National Heart Foundation and National Health and Medical Research Council of Australia. 


\section{References}

[1] A. La Gerche and D. L. Prior, "Exercise: is it possible to have too much of a good thing?" Heart Lung and Circulation, vol. 16, supplement 3, pp. S102-S104, 2007.

[2] M. Gewillig, "The fontan circulation," Heart, vol. 91, no. 6, pp. 839-846, 2005.

[3] T. Rowland, "Echocardiography and circulatory response to progressive endurance exercise," Sports Medicine, vol. 38, no. 7, pp. 541-551, 2008.

[4] André La Gerche, Andrew I. Macisaac, Andrew T. Burns et al., "Pulmonary transit of agitated contrast is associated with enhanced pulmonary vascular reserve and right ventricular function during exercise," Journal of Applied Physiology. In press.

[5] V. Stanek, P. Jebavy, J. Hurych, and J. Widimsky, "Central haemodynamics during supine exercise and pulmonary artery occlusion in normal subjects," Bulletin de Physio-Pathologie Respiratoire, vol. 9, no. 5, pp. 1203-1217, 1973.

[6] V. Stanek, J. Widimsky, S. Degre, and H. Denolin, "The lesser circulation during exercise in healthy subjects," Prog Respir Res, vol. 9, pp. 295-315, 1975.

[7] A. Giardini, A. Balducci, S. Specchia, G. Gargiulo, M. Bonvicini, and F. M. Picchio, "Effect of sildenafil on haemodynamic response to exercise and exercise capacity in fontan patients," European Heart Journal, vol. 29, no. 13, pp. 1681$1687,2008$.

[8] B. Saltin, "Hemodynamic adaptations to exercise," American Journal of Cardiology, vol. 55, no. 10, pp. 42D-47D, 1985.

[9] D. R. Bassett Jr. and E. T. Howley, "Limiting factors for maximum oxygen uptake and determinants of endurance performance," Medicine and Science in Sports and Exercise, vol. 32 , no. 1 , pp. 70-84, 2000.

[10] A. La Gerche, A. J. Taylor, and D. L. Prior, "Athlete's heart: the potential for multimodality imaging to address the critical remaining questions," JACC: Cardiovascular Imaging, vol. 2, no. 3, pp. 350-363, 2009.

[11] L. A. Wolfe, D. A. Cunningham, G. M. Davis, and H. Rosenfeld, "Relationship between maximal oxygen uptake and left ventricular function in exercise," Journal of Applied Physiology, vol. 44, no. 1, pp. 44-49, 1978.

[12] T. W. Rowland, M. Garrard, S. Marwood, M. E. Guerra, D. Roche, and V. B. Unnithan, "Myocardial performance during progressive exercise in athletic adolescent males," Medicine and Science in Sports and Exercise, vol. 41, no. 9, pp. 1721-1728, 2009.

[13] D. E. R. Warburton, M. J. Haykowsky, H. A. Quinney, D. Blackmore, K. K. Teo, and D. P. Humen, "Myocardial response to incremental exercise in endurance-trained athletes: influence of heart rate, contractility and the Frank-Starling effect," Experimental Physiology, vol. 87, no. 5, pp. 613-622, 2002.

[14] D. E. R. Warburton, N. Gledhill, V. K. Jamnik, B. Krip, and N. Card, "Induced hypervolemia, cardiac function, VO2max, and performance of elite cyclists," Medicine and Science in Sports and Exercise, vol. 31, no. 6, pp. 800-808, 1999.

[15] T. R. Kimball, W. A. Mays, P. R. Khoury, R. Mallie, and R. P. Claytor, "Echocardiographic determination of left ventricular preload, afterload, and contractility during and after exercise," Journal of Pediatrics, vol. 122, no. 6, pp. S89-S94, 1993.

[16] S. Nottin, A. Vinet, F. Stecken et al., "Central and peripheral cardiovascular adaptations during a maximal cycle exercise in boys and men," Medicine and Science in Sports and Exercise, vol. 34, no. 3, pp. 456-463, 2002.
[17] T. Rowland, A. Garrison, and A. Delulio, "Circulatory responses to progressive exercise: insights from positional differences," International Journal of Sports Medicine, vol. 24, no. 7, pp. 512-517, 2003.

[18] M. Sagiv, D. Ben-Sira, E. Goldhammer, and M. Soudry, "Left ventricular contractility and function at peak aerobic and anaerobic exercises," Medicine and Science in Sports and Exercise, vol. 32, no. 7, pp. 1197-1201, 2000.

[19] C. A. Vella and R. A. Robergs, "A review of the stroke volume response to upright exercise in healthy subjects," British Journal of Sports Medicine, vol. 39, no. 4, pp. 190-195, 2005.

[20] S. Holverda, C. T.-J. Gan, J. T. Marcus, P. E. Postmus, A. Boonstra, and A. Vonk-Noordegraaf, "Impaired stroke volume response to exercise in pulmonary arterial hypertension," Journal of the American College of Cardiology, vol. 47, no. 8, pp. 1732-1733, 2006.

[21] S. Holverda, H. Rietema, N. Westerhof et al., "Stroke volume increase to exercise in chronic obstructive pulmonary disease is limited by increased pulmonary artery pressure," Heart, vol. 95, no. 2, pp. 137-141, 2009.

[22] G.-J. Mauritz, J. T. Marcus, A. Boonstra, P. E. Postmus, N. Westerhof, and A. Vonk-Noordegraaf, "Non-invasive stroke volume assessment in patients with pulmonary arterial hypertension: left-sided data mandatory," Journal of Cardiovascular Magnetic Resonance, vol. 10, no. 1, article 51, 2008.

[23] D. Morrison, S. Sorensen, J. Caldwell et al., "The normal right ventricular response to supine exercise," Chest, vol. 82, no. 6 , pp. 686-691, 1982.

[24] A. R. Hsu, K. E. Barnholt, N. K. Grundmann, J. H. Lin, S. W. McCallum, and A. L. Friedlander, "Sildenafil improves cardiac output and exercise performance during acute hypoxia, but not normoxia," Journal of Applied Physiology, vol. 100, no. 6, pp. 2031-2040, 2006.

[25] H. A. Ghofrani, F. Reichenberger, M. G. Kohstall et al., "Sildenafil increased exercise capacity during hypoxia at low altitudes and at Mount Everest base camp: a randomized, double-blind, placebo-controlled crossover trial," Annals of Internal Medicine, vol. 141, no. 3, pp. 169-177, 2004.

[26] V. Faoro, S. Boldingh, M. Moreels et al., "Bosentan decreases pulmonary vascular resistance and improves exercise capacity in acute hypoxia," Chest, vol. 135, no. 5, pp. 1215-1222, 2009.

[27] A. W. Sheel, M. R. Edwards, G. S. Hunte, and D. C. McKenzie, "Influence of inhaled nitric oxide on gas exchange during normoxic and hypoxic exercise in highly trained cyclists," Journal of Applied Physiology, vol. 90, no. 3, pp. 926-932, 2001.

[28] S. Puwanant, M. Park, Z. B. Popović et al., "Ventricular geometry, strain, and rotational mechanics in pulmonary hypertension," Circulation, vol. 121, no. 2, pp. 259-266, 2010.

[29] E. Hart, R. Shave, N. Middleton, K. George, G. Whyte, and D. Oxborough, "Effect of preload augmentation on pulsed wave and tissue Doppler echocardiographic indices of diastolic function after a marathon," Journal of the American Society of Echocardiography, vol. 20, no. 12, pp. 1393-1399, 2007.

[30] R. G. Barr, D. A. Bluemke, F. S. Ahmed et al., "Percent emphysema, airflow obstruction, and impaired left ventricular filling," New England Journal of Medicine, vol. 362, no. 3, pp. 217-227, 2010.

[31] J. M. Norton, "Toward consistent definitions for preload and afterload," Advances in Physiology Education, vol. 25, no. 1-4, pp. 53-61, 2001.

[32] T. W. Rowland, "Circulatory responses to exercise: are we misreading fick?” Chest, vol. 127, no. 3, pp. 1023-1030, 2005. 
[33] S. E. Epstein, G. D. Beiser, M. Stampfer, B. F. Robinson, and E. Braunwald, "Characterization of the circulatory response to maximal upright exercise in normal subjects and patients with heart disease," Circulation, vol. 35, no. 6, pp. 1049-1062, 1967.

[34] J. A. L. Calbet and M. J. Joyner, "Disparity in regional and systemic circulatory capacities: do they affect the regulation of the circulation?" Acta Physiologica, vol. 199, no. 4, pp. 393406, 2010.

[35] J. J. Tolle, A. B. Waxman, T. L. Van Horn, P. P. Pappagianopoulos, and D. M. Systrom, "Exercise-induced pulmonary arterial hypertension," Circulation, vol. 118, no. 21, pp. 2183-2189, 2008.

[36] S. M. Proudman, W. M. Stevens, J. Sahhar, and D. Celermajer, "Pulmonary arterial hypertension in systemic sclerosis: the need for early detection and treatment," Internal Medicine Journal, vol. 37, no. 7, pp. 485-494, 2007.

[37] P. Argiento, N. Chesler, M. Mulè et al., "Exercise stress echocardiography for the study of the pulmonary circulation," European Respiratory Journal, vol. 35, no. 6, pp. 1273-1278, 2010.

[38] E. Bossone, M. Rubenfire, D. S. Bach, M. Ricciardi, and W. F. Armstrong, "Range of tricuspid regurgitation velocity at rest and during exercise in normal adult men: implications for the diagnosis of pulmonary hypertension," Journal of the American College of Cardiology, vol. 33, no. 6, pp. 1662-1666, 1999.

[39] C. M. Bidart, A. E. Abbas, J. M. Parish, H. P. Chaliki, C. A. Moreno, and S. J. Lester, "The noninvasive evaluation of exercise-induced changes in pulmonary artery pressure and pulmonary vascular resistance," Journal of the American Society of Echocardiography, vol. 20, no. 3, pp. 270-275, 2007.

[40] M. K. Stickland, R. C. Welsh, M. J. Haykowsky et al., "Effect of acute increases in pulmonary vascular pressures on exercise pulmonary gas exchange," Journal of Applied Physiology, vol. 100, no. 6, pp. 1910-1917, 2006.

[41] J. T. Reeves, B. M. Groves, A. Cymerman et al., "Operation Everest II: cardiac filling pressures during cycle exercise at sea level," Respiration Physiology, vol. 80, no. 2-3, pp. 147-154, 1990.

[42] G. Kovacs, A. Berghold, S. Scheidl, and H. Olschewski, "Pulmonary arterial pressure during rest and exercise in healthy subjects: a systematic review," European Respiratory Journal, vol. 34, no. 4, pp. 888-894, 2009.

[43] C. A. Dawson, "Role of pulmonary vasomotion in physiology of the lung," Physiological Reviews, vol. 64, no. 2, pp. 544-616, 1984.

[44] D. Burkhoff, I. Mirsky, and H. Suga, "Assessment of systolic and diastolic ventricular properties via pressure-volume analysis: a guide for clinical, translational, and basic researchers," American Journal of Physiology, vol. 289, no. 2, pp. H501H512, 2005.

[45] E. V. Buechel, T. Kaiser, C. Jackson, A. Schmitz, and C. J. Kellenberger, "Normal right- and left ventricular volumes and myocardial mass in children measured by steady state free precession cardiovascular magnetic resonance," Journal of Cardiovascular Magnetic Resonance, vol. 11, no. 1, p. 19, 2009.

[46] M. J. Faber, M. Dalinghaus, I. M. Lankhuizen et al., "Right and left ventricular function after chronic pulmonary artery banding in rats assessed with biventricular pressure-volume loops," American Journal of Physiology, vol. 291, no. 4, pp. H1580-H1586, 2006.

[47] S. Brimioulle, P. Wauthy, P. Ewalenko et al., "Single-beat estimation of right ventricular end-systolic pressure-volume relationship," American Journal of Physiology, vol. 284, no. 5, pp. H1625-H1630, 2003.
[48] W. MacNee, "Pathophysiology of cor pulmonale in chronic obstructive pulmonary disease," American Journal of Respiratory and Critical Care Medicine, vol. 150, no. 3, pp. 833-852, 1994.

[49] A. La Gerche, K. A. Connelly, D. J. Mooney, A. I. MacIsaac, and D. L. Prior, "Biochemical and functional abnormalities of left and right ventricular function after ultra-endurance exercise," Heart, vol. 94, no. 7, pp. 860-866, 2008.

[50] P. S. Douglas, M. L. O’Toole, W. D. B. Hiller, and N. Reichek, "Different effects of prolonged exercise on the right and left ventricles," Journal of the American College of Cardiology, vol. 15 , no. 1, pp. 64-69, 1990.

[51] A. J. Camm and L. Fei, "Chronotropic incompetence-part I: normal regulation of the heart rate," Clinical Cardiology, vol. 19, no. 5, pp. 424-428, 1996.

[52] H. Ohuchi, "Cardiopulmonary response to exercise in patients with the fontan circulation," Cardiology in the Young, vol. 15, supplement 3, pp. 39-44, 2005.

[53] E. S. Larsson, B. O. Eriksson, and R. Sixt, "Decreased lung function and exercise capacity in fontan patients. A long-term follow-up," Scandinavian Cardiovascular Journal, vol. 37, no. 1, pp. 58-63, 2003.

[54] M. Gewillig, S. C. Brown, B. Eyskens et al., "The fontan circulation: who controls cardiac output?" Interactive Cardiovascular and Thoracic Surgery, vol. 10, no. 3, pp. 428-433, 2010.

[55] H. Senzaki, S. Masutani, H. Ishido et al., "Cardiac rest and reserve function in patients with fontan circulation," Journal of the American College of Cardiology, vol. 47, no. 12, pp. 2528$2535,2006$.

[56] H. Senzaki, S. Masutani, J. Kobayashi et al., "Ventricular afterload and ventricular work in fontan circulation: comparison with normal two-ventricle circulation and single-ventricle circulation with blalock-taussig shunts," Circulation, vol. 105, no. 24, pp. 2885-2892, 2002.

[57] D. Robbers-Visser, D. Jan ten Harkel, L. Kapusta et al., "Usefulness of cardiac magnetic resonance imaging combined with low-dose dobutamine stress to detect an abnormal ventricular stress response in children and young adults after fontan operation at young age," American Journal of Cardiology, vol. 101, no. 11, pp. 1657-1662, 2008.

[58] D. De Wolf, B. Suys, H. Verhaaren, D. Matthys, and Y. Taeymans, "Low-dose dobutamine stress echocardiography in children and young adults," American Journal of Cardiology, vol. 81, no. 7, pp. 895-901, 1998.

[59] T. Oosterhof, I. I. Tulevski, A. A. W. Roest et al., "Disparity between dobutamine stress and physical exercise magnetic resonance imaging in patients with an intra-atrial correction for transposition of the great arteries," Journal of Cardiovascular Magnetic Resonance, vol. 7, no. 2, pp. 383-389, 2005.

[60] B. H. Goldstein, C. E. Connor, L. Gooding, and A. P. Rocchini, "Relation of systemic venous return, pulmonary vascular resistance, and diastolic dysfunction to exercise capacity in patients with single ventricle receiving fontan palliation," American Journal of Cardiology, vol. 105, no. 8, pp. 1169-1175, 2010.

[61] M. I. Burgess, C. Jenkins, J. E. Sharman, and T. H. Marwick, "Diastolic stress echocardiography: Hemodynamic validation and clinical significance of estimation of ventricular filling pressure with exercise," Journal of the American College of Cardiology, vol. 47, no. 9, pp. 1891-1900, 2006. 
[62] D. R. Talreja, R. A. Nishimura, and J. K. Oh, "Estimation of left ventricular filling pressure with exercise by Doppler echocardiography in patients with normal systolic function: a simultaneous echocardiographic-cardiac catheterization study," Journal of the American Society of Echocardiography, vol. 20, no. 5, pp. 477-479, 2007.

[63] H. Ohuchi, H. Takasugi, H. Ohashi et al., "Abnormalities of neurohormonal and cardiac autonomic nervous activities relate poorly to functional status in fontan patients," Circulation, vol. 110, no. 17, pp. 2601-2608, 2004.

[64] T. Akagi, L. N. Benson, M. Green et al., "Ventricular performance before and after fontan repair for univentricular atrioventricular connection: angiographic and radionuclide assessment," Journal of the American College of Cardiology, vol. 20, no. 4, pp. 920-926, 1992.

[65] A. A. Kouatli, J. A. Garcia, T. M. Zellers, E. M. Weinstein, and L. Mahony, "Enalapril does not enhance exercise capacity in patients after fontan procedure," Circulation, vol. 96, no. 5, pp. 1507-1512, 1997.

[66] G. Barber, T. Di Sessa, J. S. Child et al., "Hemodynamic responses to isolated increments in heart rate by atrial pacing after a fontan procedure," American Heart Journal, vol. 115, no. 4, pp. 837-841, 1988.

[67] M. H. Gewillig, U. R. Lundstrom, C. Bull, R. K. H. Wyse, and J. E. Deanfield, "Exercise responses in patients with congenital heart disease after fontan repair: patterns and determinants of performance," Journal of the American College of Cardiology, vol. 15, no. 6, pp. 1424-1432, 1990.

[68] T. Takken, M. H. P. Tacken, A. C. Blank, E. H. Hulzebos, J. L. M. Strengers, and P. J. M. Helders, "Exercise limitation in patients with fontan circulation: a review," Journal of Cardiovascular Medicine, vol. 8, no. 10, pp. 775-781, 2007.

[69] T. M. Zellers, D. J. Driscoll, C. D. Mottram, F. J. Puga, H. V. Schaff, and G. K. Danielson, "Exercise tolerance and cardiorespiratory response to exercise before and after the fontan operation," Mayo Clinic Proceedings, vol. 64, no. 12, pp. 1489-1497, 1989.

[70] R. G. S. Cortes, G. Satomi, M. Yoshigi, and K. Momma, "Maximal hemodynamic response after the fontan procedure: doppler evaluation during the treadmill test," Pediatric Cardiology, vol. 15, no. 4, pp. 170-177, 1994.

[71] A. Zajc, L. Tomkiewicz, P. Podolec, W. Tracz, and E. Malec, "Cardiorespiratory response to exercise in children after modified fontan operation," Scandinavian Cardiovascular Journal, vol. 36, no. 2, pp. 80-85, 2002.

[72] M. Gewillig, R. K. Wyse, M. R. De Leval, and J. E. Deanfield, "Early and late arrhythmias after the fontan operation: predisposing factors and clinical consequences," British Heart Journal, vol. 67, no. 1, pp. 72-79, 1992.

[73] M. I. Cohen, L. A. Rhodes, G. Wernovsky, J. W. Gaynor, T. L. Spray, and J. Rychik, "Atrial pacing: an alternative treatment for protein-losing enteropathy after the fontan operation," Journal of Thoracic and Cardiovascular Surgery, vol. 121, no. 3, pp. 582-583, 2001. 


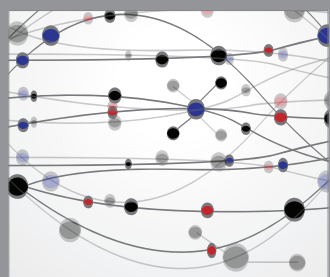

The Scientific World Journal
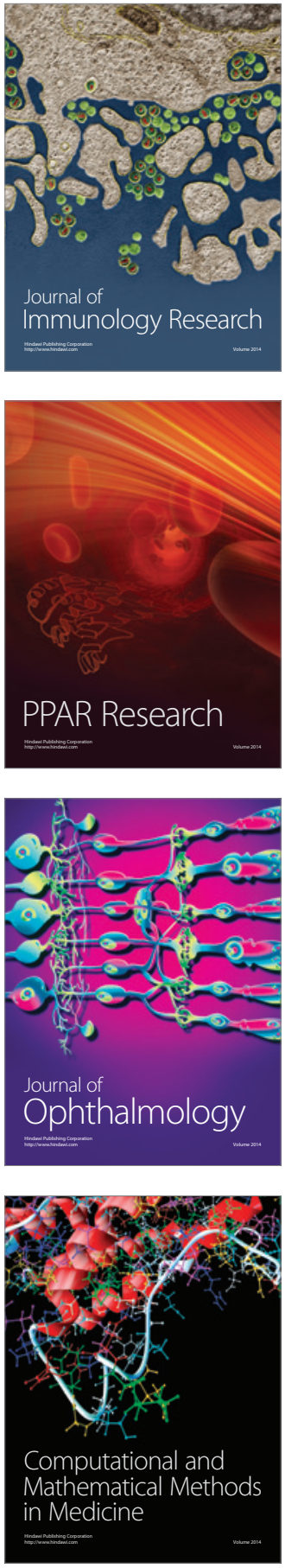

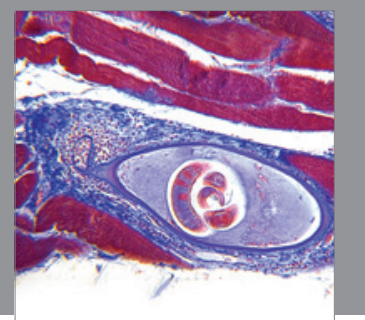

Gastroenterology

Research and Practice
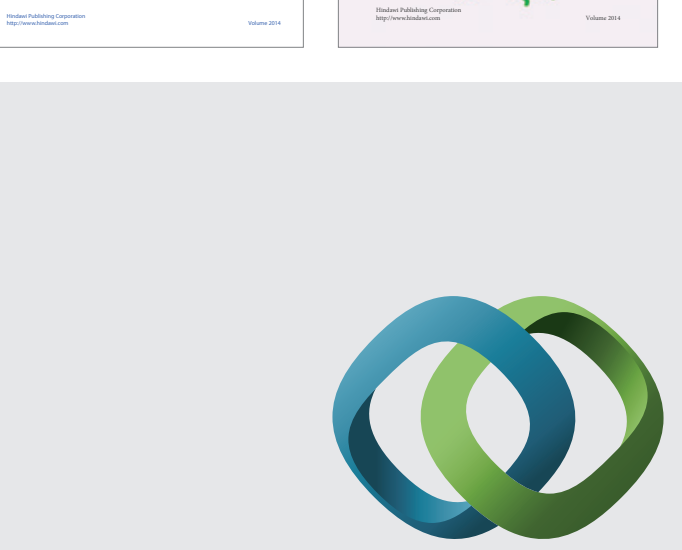

\section{Hindawi}

Submit your manuscripts at

http://www.hindawi.com
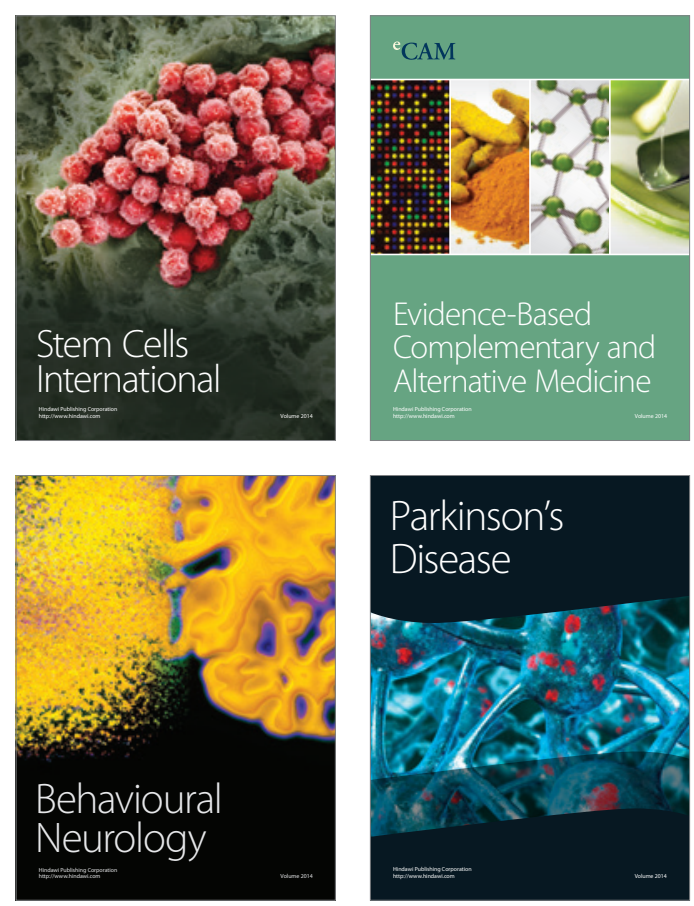

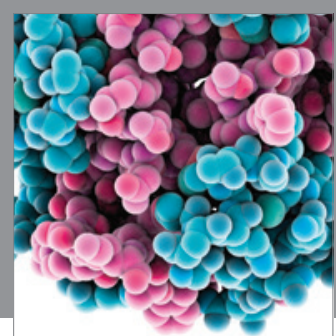

Journal of
Diabetes Research

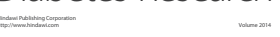

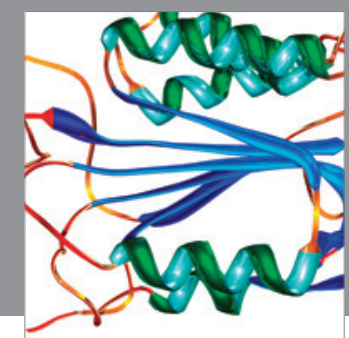

Disease Markers
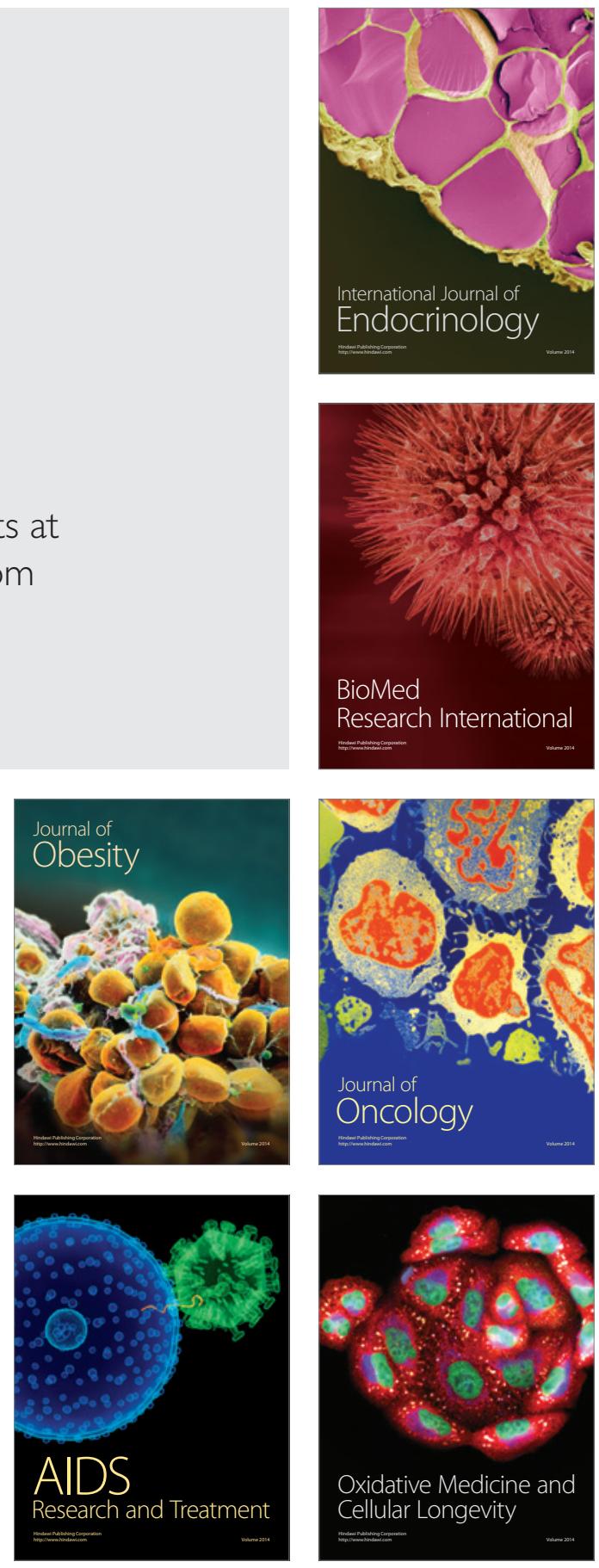